\title{
Study on the Dependence of Reverse Simulation for Identifying a Pollutant Source on Grid Resolution and Filter Width in Cavity Flow
}

\author{
Satoshi Abe, ${ }^{1}$ Shinsuke Kato, ${ }^{2}$ Fujihiro Hamba, ${ }^{2}$ \\ and Daisuke Kitazawa ${ }^{2}$ \\ ${ }^{1}$ Graduate School of Engineering, The University of Tokyo, Tokyo 113-8656, Japan \\ ${ }^{2}$ Institute of Industrial Science, The University of Tokyo, Tokyo 153-8505, Japan \\ Correspondence should be addressed to Satoshi Abe, abe.satoshi@jaea.go.jp
}

Received 16 December 2011; Revised 2 March 2012; Accepted 3 March 2012

Academic Editor: Fu-Yun Zhao

Copyright (C) 2012 Satoshi Abe et al. This is an open access article distributed under the Creative Commons Attribution License, which permits unrestricted use, distribution, and reproduction in any medium, provided the original work is properly cited.

When a hazardous substance is diffused, it is necessary to identify the pollutant source and respond immediately. However, there are many cases in which damage is caused without a clear understanding of where the pollutant source is located. There are three groups of identifying pollutant source information (Liu and Zhai, 2007): the probability method, forward method, and backward method. In our previous study, we proposed reverse simulation, which is categorized as a backward method (Abe and Kato, 2011). Numerical instability by negative diffusion is a principal problem in the backward method. In order to improve the problem, we applied a low-pass filter operation to the concentration flux in the RANS analysis. The simulation secured the numerical stability. However, reverse simulation accuracy is expected to depend on the grid resolution and filter width. In this paper, we introduce reverse simulation results in cavity flow. In particular, we survey the dependence of reverse simulation accuracy on the grid resolution and filter width. Moreover, we discuss the dependence of reverse simulation on the grid resolution and filter width with a one-dimensional diffusion equation. As a result, we found that the simulated negative diffusion varies greatly among the grid resolution and filter width.

\section{Introduction}

When a hazardous substance is diffused, it is necessary to identify the source of the pollutant and respond immediately, for example, by cleaning up and evacuating people from the area. However, there are many cases in which damage is caused without a clear understanding of where the pollutant source is located. Many lives have been lost as a result of accidental pollution from power stations and factories (e.g., Chernobyl in 1986, Bopal in 1984). 
In recent years, some researchers have studied techniques of identifying pollutant sources either in the groundwater or air. The techniques are separated into three groups: probability method, forward method, and backward method [1].

Probability methods determine the pollutant source information with probability objective functions. Wagner developed nonlinear maximum likelihood estimation for simultaneous model parameter estimation and source characterization [2]. The method combines groundwater flow and contaminant transport simulation. Woodbury et al. applied minimum relative entropy theory in order to recover the release history in groundwater flow [3]. Neupauer et al. developed an adjoint method as a formal framework for predicting groundwater contaminant source location and travel-time probabilities [4]. Sohn et al. worked on rapidly locating and characterizing air pollutant releases and presented an approach for estimating the source locations, the amounts released, and the dispersion characteristics of indoor airborne pollutants [5]. They proposed a two-stage Bayesian data interpretation approach.

The forward method identifies the pollutant source by trial-and-error algorithm. The processes describe the match degree of measured and simulated results and can be used to estimate the pollutant sources. Gorelick et al. applied minimizing normalized residual for identifying the source and magnitude of groundwater pollutants in some two-dimensional cases [6]. Alapati and Kabala applied the least squares function to predict the source release history in a one-dimensional underground water problem [7]. In the forward method, there are ways of applying the Tikhonov regularization method [8], which is the most commonly employed method for an ill-posed problem. Skaggs and Kabala applied the regularization method to recover the release history in one-dimensional simulation [9]. In atmospheric gaseous dispersion, Seibert et al. used the Tikhonov regularization method [10], which is the most useful method for an ill-posed problem to derive the source history from ambient concentration measurement. These forward methods are good tools for identifying source information. However, every forward method requires many calculations.

The backward method is the solution to the transport equation in negative time with the end status as the input condition. The most important problem in the backward method is the numerical instability from negative diffusion. Generically, CFD needs to define the location with a finite grid resolution, and there are high wave numbers that cannot be resolved in CFD. Here, wave number is expressed as " $k=2 \pi / \lambda$," where $\lambda$ is the wave length. The high wave number region has rounding error. Meanwhile, the numerical solution converges on a large space gradient when analyzing negative diffusion on CFD. This means that the high wave number component amplifies more quickly than that of the resolved low wave number region. Therefore, the numerical simulation breaks down due to the rounding errors in the high wave number region [11].

The most useful method for improving numerical instability in backward method is the quasi-reversible (QR) solution, which is developed by Skaggs and Kabala [12]. The QR method adds a term to the diffusion term to improve the numerical stability. Equation (1.1) shows the one-dimensional government equation in the QR method

$$
\frac{\partial C}{\partial t}=\Gamma \frac{\partial^{2} C}{\partial x^{2}}+\varepsilon \frac{\partial^{4} C}{\partial x^{4}}
$$

$\Gamma$ represents the diffusion coefficient, and $\varepsilon$ represents the stabilization coefficient. This equation can solve with a negative time step. In Skaggs and Kabala's research, the method 
was used to recover the time history and spatial distribution of a groundwater contaminant plume.

In our previous study, we introduced reverse simulation [13, 14], which was categorized as a backward method. The method applies a lowpass filter operation to improve the numerical stability $(1.2)$

$$
\begin{gathered}
\bar{F}(x)=\int_{-\infty}^{\infty} G(r) F(x-r) d r, \\
G(r)=\sqrt{\frac{6}{\pi \Delta^{2}}} \exp \frac{-6 r^{2}}{\Delta^{2}},
\end{gathered}
$$

$F$ is a physical quantity that is applied in the filter operation. The filtered physical quantity is shown with an overbar, and $\Delta$ is the filter width.

The most important problem when carrying out a transport equation with negative time advancing in CFD is the numerical instability by analyzing negative diffusion. We made a pass at applying the filter operation to a concentration field (1.3) in Reynolds averaged numerical simulation (RANS) analysis [13]

$$
F(x)=C(x),
$$

$C$ means the concentration. This method improved the numerical stability, but there was a big problem with the analysis results. The concentration distribution spread diffusely in an analytical domain. This means that it is difficult to identify a pollutant source with reverse simulation applying a filter operation to a concentration field. Therefore, we developed a reverse simulation. We made a pass at applying the filter operation to a concentration flux (1.5) to improve the numerical stability and solve the above problem [14]

$$
\begin{gathered}
F_{i}(x)=-\left\langle u_{i}^{\prime} c^{\prime}\right\rangle=\frac{v_{t}}{\sigma_{3}} \frac{\partial C(x)}{\partial x_{i}}, \\
F_{i}(x)=\frac{\partial C(x)}{\partial x_{i}} .
\end{gathered}
$$

In (1.3), $-\left\langle u_{i}^{\prime} c^{\prime}\right\rangle$ is turbulent concentration flux. Equation (1.4) means gradient diffusion approximation, $v_{t}$ is the coefficient of turbulent diffusion, and $\sigma_{3}$ is the turbulent Schmidt number. In RANS analysis, it was necessary to apply the filter operation to (1.4) strictly. However, $v_{t}$ is dependent on the position. This makes the filter operation cumbersome and complicated. Therefore, we applied the filter operation to the differential part of only (1.5) approximately. The simulation secured the numerical stability and solved the problem of concentration spreading diffusely. However, the reverse simulation accuracy is expected to depend on the grid resolution and filter width.

In this paper, we survey the dependence of reverse simulation accuracy on the grid resolution and filter width in cavity flow, which forms a greatly bent and circulating flow. The analysis can be carried out with three different grid resolutions. Each grid resolution has three different filter widths. A total of 9 cases were analyzed in this study. In addition, we discuss the dependence of reverse simulation on gird resolution and filter width with a one-dimensional diffusion equation. 


\section{Analysis Method}

\subsection{RANS Government Equation}

In the present study, the equations formed to govern incompressible flow in RANS analysis were mass conservation flow equation (2.1), the Navier-Stokes equation (2.2), turbulent kinetic energy $(K)$ equation (2.3), turbulent dissipation $(\varepsilon)$ equation (2.4), and concentration $(C)$ transport equation (2.5). The concentration is passive scalar, assuming that the flow field has no influence. The turbulence model in this paper is a two-equation Kato-Launder-type model [15]

$$
\begin{aligned}
& \frac{\partial U_{i}}{\partial x_{i}}=0, \\
& \frac{\partial U_{i}}{\partial t}+U_{j} \frac{\partial U_{i}}{\partial x_{j}}=-\frac{1}{\rho} \frac{\partial}{\partial x_{i}}\left(P+\frac{2}{3} K\right)+\frac{\partial}{\partial x_{j}}\left\{v_{t}\left(\frac{\partial U_{i}}{\partial x_{j}}+\frac{\partial U_{j}}{\partial x_{i}}\right)\right\} \\
& \frac{\partial K}{\partial t}+U_{i} \frac{\partial K}{\partial x_{i}}=\frac{\partial}{\partial x_{i}}\left(\frac{v_{t}}{\sigma_{1}} \frac{\partial K}{\partial x_{i}}\right)+P_{K}-\varepsilon, \\
& \frac{\partial \varepsilon}{\partial t}+U_{i} \frac{\partial \varepsilon}{\partial x_{i}}=\frac{\partial}{\partial x_{i}}\left(\frac{v_{t}}{\sigma_{2}} \frac{\partial \varepsilon}{\partial x_{i}}\right)+\frac{\varepsilon}{K} C_{1} P_{k}-C_{2} \frac{\varepsilon^{2}}{K}, \\
& \frac{\partial C}{\partial t}+U_{i} \frac{\partial C}{\partial x_{i}}=\frac{\partial}{\partial x_{i}}\left(\frac{v_{t}}{\sigma_{3}} \frac{\partial C}{\partial x_{i}}\right) \\
& v_{t}=C_{\mu} \frac{K^{2}}{\varepsilon} \\
& P_{k}=v_{t} S \Omega \text {, } \\
& S=\sqrt{\frac{1}{2}\left(\frac{\partial U_{i}}{\partial x_{j}}+\frac{\partial U_{j}}{\partial x_{i}}\right)^{2}}, \\
& \Omega=\sqrt{\frac{1}{2}\left(\frac{\partial U_{i}}{\partial x_{j}}+\frac{\partial U_{j}}{\partial x_{i}}\right)^{2}}, \\
& \sigma_{1}=1.0, \quad \sigma_{2}=1.3, \quad \sigma_{3}=1.0, \quad C_{\mu}=0.09, \quad C_{1}=1.44, \quad C_{2}=1.92 \text {. }
\end{aligned}
$$

The process of negative time advancing in the transport equation is equivalent to that of positive time advancing with negative time convection and diffusion (2.7)

$$
\frac{\partial C}{\partial t}-U_{i} \frac{\partial C}{\partial x_{i}}=-\frac{\partial}{\partial x_{i}}\left(\frac{v_{t}}{\sigma_{3}} \frac{\partial C}{\partial x_{i}}\right)
$$

But the equation is unstable with time advancing. Overall, in forward analysis, the diffusion term has the effect of improving the numerical stability because the diffusion coefficients $\left(v_{t}\right)$ are a positive value. However, in the backward method, the coefficients are a negative value. A diffusion term that has a negative coefficient increases the intensity of the high wave 
number region and destabilizes the numerical simulation. For this, we apply lowpass filter operation to (1.2) the concentration flux (1.5). Equation (2.7) is converted to (2.8) by the filter operation

$$
\frac{\partial C}{\partial t}-U_{i} \frac{\partial C}{\partial x_{i}}=-\frac{\partial}{\partial x_{i}}\left(\frac{v_{t}}{\sigma_{3}} \frac{\overline{\partial C}}{\partial x_{i}}\right)
$$

The idea is to simulate the negative diffusion played by the low wave number region and cut that by the high wave number region. The lowpass filter operation to the concentration flux is clearly beneficial in [14].

In the present stage, flow fluctuations are not considered. Essentially, it is good to consider the flow fluctuation because the air flow characteristics are unsteady due to fluctuation in both speed and direction. However, it is necessary for treating the unsteady flow to solve the Navier-Stokes equation (2.2) in negative time (inverse analysis) or keep full-time and space-series data. The former is impossible, because it is necessary to couple the Navier-Stokes equation to the mass conservation equation (2.1). The latter requires huge memory to store the time-series data. In addition, our purpose in this paper is to survey and discuss the dependence of reverse simulation on the grid resolution and filter width. In consideration of these circumstances, we treat the steady flow in the forward and reverse analyses.

\subsection{The Numerical Discretization, Simulation Method, and Analysis Model}

Regarding a lowpass filter operation for reverse numerical calculations, the Gaussian filter is given the Taylor expansion and discretization

$$
\begin{gathered}
\bar{F}(x)=F(x)+\frac{F^{\prime \prime}(x)}{2} \int_{-\infty}^{\infty} r^{2} G(r) d r+\frac{F^{(4)}(x)}{24} \int_{-\infty}^{\infty} r^{4} G(r) d r+\cdots \\
\bar{F}_{m}=F_{m}+\frac{\Delta^{2}}{24} \frac{F_{m-1}-2 F_{m}+F_{m+1}}{\Delta x^{2}}
\end{gathered}
$$

Equation (2.8) is expressed as (2.11) when using (2.10)

$$
\frac{\partial C}{\partial t}-U_{i} \frac{\partial C}{\partial x_{i}}=-\frac{\partial}{\partial x_{i}} \frac{v_{t}}{\sigma_{3}}\left\{\frac{\partial C}{\partial x_{i}}+\frac{\Delta^{2}}{24} \frac{\partial^{2}}{\partial x_{i}^{2}}\left(\frac{\partial C}{\partial x_{i}}\right)\right\} .
$$

The second term of the right-hand side of (2.11) is able to improve the numerical stability in backward analysis. Table 1 shows a summary of the parameters used in the numerical simulation. For spatial discretization in all governing equations, a second-order accurate central difference scheme is used for the convection terms and diffusion terms. In addition, when the concentration is below 0 , it is replaced as 0 (clipping method). For time integration, the convection and diffusion terms in the flow field are discretized using the AdamsBashforth schemes. Also, the convection and diffusion in the scalar fields (concentration, 
Table 1: Numerical simulation parameters.

\begin{tabular}{ll}
\hline Turbulent model & Kato-Launder k-åmodel \\
Navier-Stokes equation & Convection term: 2nd-order central difference scheme \\
& Diffusion term: 2nd-order central difference scheme \\
& Convection term: 2nd-order central difference scheme \\
Scalar transport equation $(K, \varepsilon, C)$ & Diffusion term: 2nd-order central difference scheme \\
& Convection term: Adams-Bashforth method \\
Time advancing & Diffusion term: Adams-Bashforth method \\
Algorithm & ABMAC type \\
\hline
\end{tabular}

Table 2: Numerical grids and filter width in each case.

\begin{tabular}{|c|c|c|}
\hline \multicolumn{2}{|c|}{ Analysis case } & Analysis condition (numerical grid resolution and filter width) \\
\hline Case 1 & $\begin{array}{l}\text { Case } 1-1 \\
\text { Case } 1-2 \\
\text { Case } 1-3\end{array}$ & $\begin{array}{l}\Delta x / H_{b}=0.2, \Delta y / H_{b}=0.2, \Delta z / H_{b}=0.1, \Delta=10.0 \ddot{A} x_{i} \\
\Delta x / H_{b}=0.2, \Delta y / H_{b}=0.2, \Delta z / H_{b}=0.1, \Delta=15.0 \ddot{A} x_{i} \\
\Delta x / H_{b}=0.2, \Delta y / H_{b}=0.2, \Delta z / H_{b}=0.1, \Delta=20.0 \ddot{A} x_{i}\end{array}$ \\
\hline Case 2 & $\begin{array}{l}\text { Case } 2-1 \\
\text { Case } 2-2 \\
\text { Case } 2-3\end{array}$ & $\begin{array}{l}\Delta x / H_{b}=0.1, \Delta y / H_{b}=0.1, \Delta z / H_{b}=0.05, \Delta=10.0 \ddot{A} x_{i} \\
\Delta x / H_{b}=0.1, \Delta y / H_{b}=0.1, \Delta z / H_{b}=0.05, \Delta=15.0 \ddot{A} x_{i} \\
\Delta x / H_{b}=0.1, \Delta y / H_{b}=0.1, \Delta z / H_{b}=0.05, \Delta=20.0 \ddot{A} x_{i}\end{array}$ \\
\hline Case 3 & $\begin{array}{l}\text { Case } 3-1 \\
\text { Case } 3-2 \\
\text { Case } 3-3\end{array}$ & $\begin{array}{l}\Delta x / H_{b}=0.05, \Delta y / H_{b}=0.05, \Delta z / H_{b}=0.05, \Delta=10.0 \ddot{A} x_{i} \\
\Delta x / H_{b}=0.05, \Delta y / H_{b}=0.05, \Delta z / H_{b}=0.05, \Delta=15.0 \ddot{A} x_{i} \\
\Delta x / H_{b}=0.05, \Delta y / H_{b}=0.05, \Delta z / H_{b}=0.05, \Delta=20.0 \ddot{A} x_{i}\end{array}$ \\
\hline
\end{tabular}

turbulent kinetic energy and turbulent dissipation fields) are discretized using the AdamsBashforth schemes. In terms of the Navier-Stokes equation and mass conservation equation, the coupling algorithm of the velocity and pressure is based on the ABMAC type [16].

Regarding the grid number and filter width, Table 2 shows the analysis grid resolutions and filter width in each case. Case 1 is the coarsest grid resolution, and case 3 is the finest grid resolution in all cases. Furthermore, we set three different filter widths in each grid resolution.

The domain size is $2.0 H_{b}, 2.0 H_{b}$, and $5.0 H_{b}$ (streamwise, spanwise, and vertical, resp.). Here, $H_{b}$ is the cavity height. The cavity size is $1.0 H_{b}, 2.0 H_{b}$, and $1.0 H_{b}$ (Figure 1). The streamwise, spanwise, and vertical directions are $x\left(x_{1}\right), y\left(x_{2}\right)$, and $z\left(x_{3}\right)$, respectively. Regarding the boundary conditions, in these analyses, the periodic boundary condition is imposed on the streamwise direction for velocity field, turbulent kinetic energy, and turbulent dissipation. The Neumann condition is imposed on the top boundary for all physical quantities. The boundary conditions for bottom and walls are given by the generalized loglaw for the velocity field and the Neumann conditions for turbulent kinetic energy, turbulent dissipation, and concentration field. In addition, the Neumann conditions are applied to all boundary conditions for the filter operation.

Figure 2 shows the normalized analysis time and the normalized emission time of concentration $\left(t U_{b} / H_{b}\right)$. The forward and reverse analysis times are, respectively, from 0 to 100 and from 100 to 200 , which are normalized by $H_{b}$ and $U_{b}$. $U_{b}$ is the velocity at $H_{b}$. As we developed a method of identifying the pollutant source, it is necessary to identify the elapsed time since emission in addition to the source location. But this time, we assume that the elapsed time is known. The emission time is from 0 to 20 . The source point is $0.2 H_{b}$, below the head of the cavity and $0.2 \mathrm{H}_{b}$ downstream of the upper part of the cavity. 


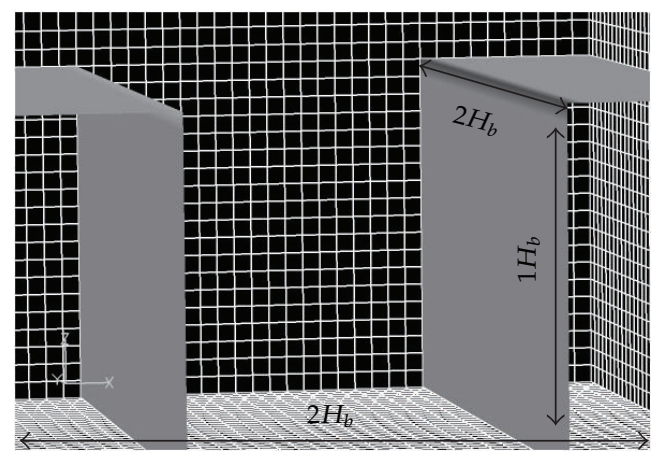

Figure 1: Analysis domain: the domain size of streamwise, span, and vertical directions is $2.0 H_{b}, 2.0 H_{b}$, and $5.5 H_{b}$, respectively. $H_{b}$ refers to the cavity height.

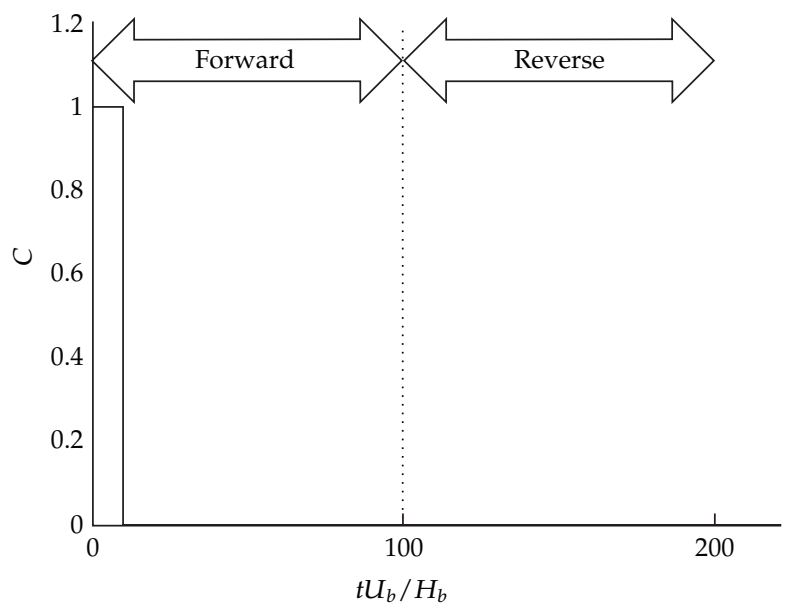

Figure 2: Emission time and analysis time, forward analysis time: 0 100, reverse analysis time: 100 200. $U_{b}$ refers to the velocity at $H_{b}$.

\section{Analysis Results}

\subsection{Forward Analysis Results}

Figure 3 shows the vector of the velocity fields in the cavity. In each analysis case, the velocity fields form circulatory flows, and all cases are similar for the stagnation point. All analysis cases have almost the same velocity field.

Figure 4 shows the results of forward analysis $\left(t U_{b} / H_{b}=100\right)$. The results are to show the input conditions for reverse simulation. These contours are normalized by the peak concentration in each case. In Case 1, the location of peak concentration slightly shifts to the center of the bottom due to a difference of grid resolution. However, the concentration distributions of all cases are similar overall. With the forward analysis results, reverse simulations are carried out. 


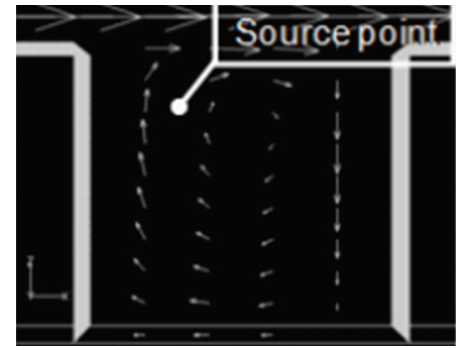

(a)

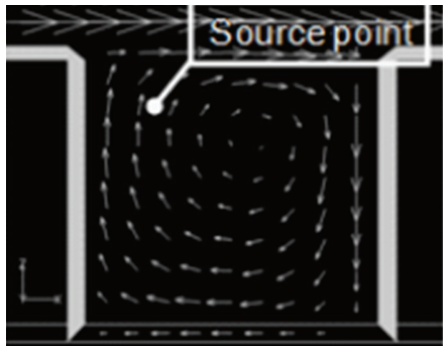

(b)

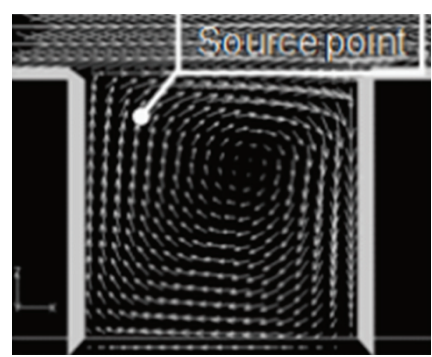

(c)

Figure 3: Side view of velocity vector: (a) Case 1, (b) Case 2, and (c) Case 3.

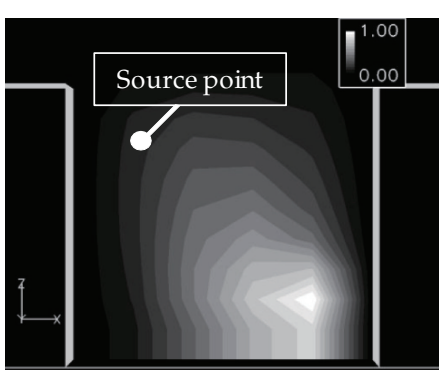

(a)

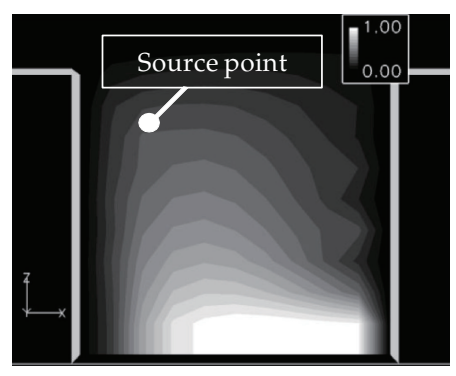

(b)

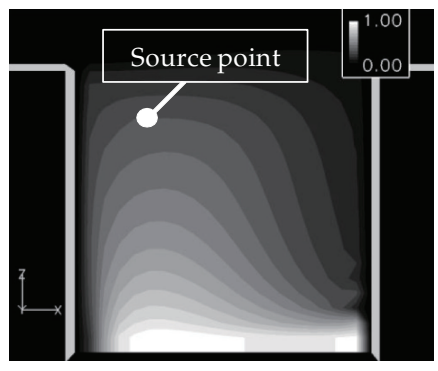

(c)

Figure 4: Input conditions for reverse simulation $\left(t U_{b} / H_{b}=100\right)$ : (a) Case 1, (b) Case 2, and (c) Case 3.

\subsection{Reverse Analysis Results}

\subsubsection{The Influence of Grid Resolution}

Figure 5 shows the results of reverse simulations for three different grid resolutions $\left(t U_{b} / H_{b}=200\right)$. These distributions are normalized by the peak concentrations of each case at $t U_{b} / H_{b}=100$. In each case, numerical instabilities are improved by the filter operation.

However, regarding Case 1-1, which has the coarsest grid resolution (Case 1-1), the concentration distribution spreads widely over the whole cavity. In addition, the high concentration location is very far from the source point as the initial condition of forward analysis because the lowpass filter effect is too strong. In other words, the numerical grid resolution of Case 1 is too rough to carry out reverse simulation and it is difficult to identify a pollutant source when using the grid resolution. Meanwhile, high concentration parts of Case 2-1 and Case 3-1 are clearer than that of Case 1-1. In particular, that of Case 3-1 is the clearest in these analyses.

To identify the pollutant source, we consider the high concentration distributions. Figure 6 shows distributions of over 80 percent of peak concentration in Case 1-1, Case 21 , and Case 3-1, respectively. These figures are normalized by the peak concentrations in each case at $t U_{b} / H_{b}=100$. The location of Case 1-1 is not appropriate, because the filter effect is too strong. The peak locations of Case 2-1 and Case 3-1 are very near to the source point as the initial condition of the forward analysis, especially the result of Case 3-1. Moreover, the grid resolution is concerned with the peak values. The values of Case 1-1 and Case 2-1 are lower than those at $t U_{b} / H_{b}=100$, and the peak value of Case 3-1 is higher than that at $t U_{b} / H_{b}=100$. These results suggest that reverse simulation can create negative diffusion played by a certain level of wave number region. 


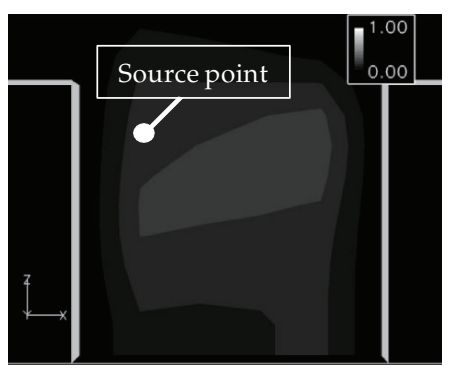

(a)

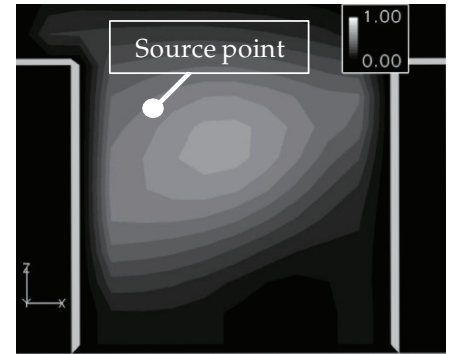

(b)

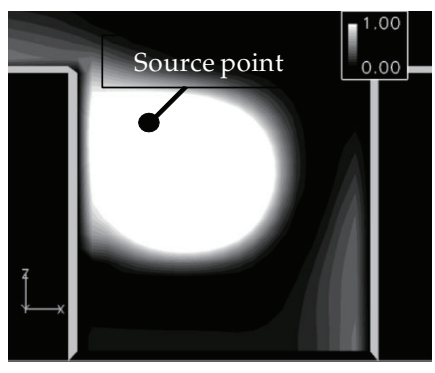

(c)

Figure 5: Reverse analysis results $\left(t U_{b} / H_{b}=200\right)$ : (a) Case 1-1, (b) Case 2-1, and (c) Case 3-1.

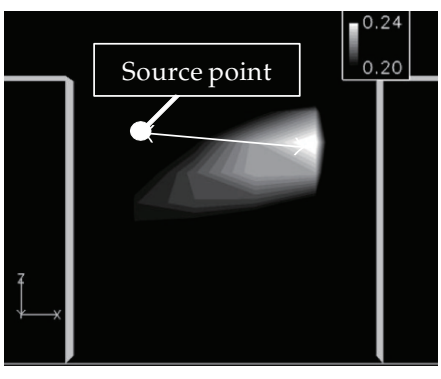

(a)

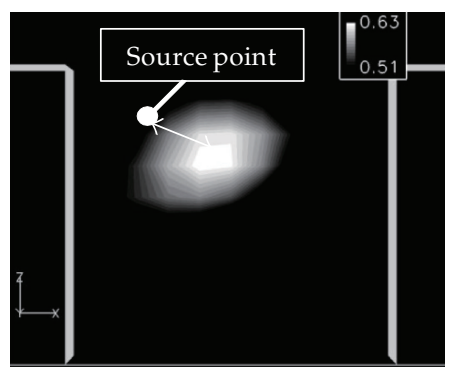

(b)

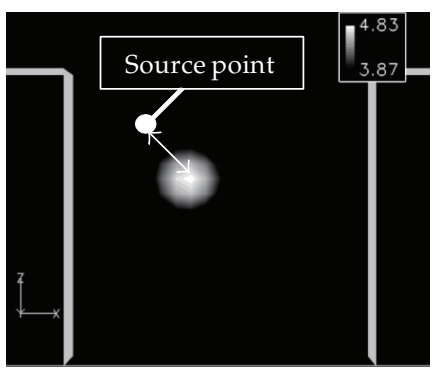

(c)

Figure 6: Peak concentration $\left(t U_{b} / H_{b}=200\right)$ : (a) Case 1-1, (b) Case 2-1, and (c) Case 3-1.

\subsubsection{The Influence of Filter Width}

Figure 7 shows the results of Case 3-2 and Case 3-3, respectively. Comparing Case 3-1 with Case 3-2 and Case 3-3 reveals that the high concentration parts wash out as the filter widths are larger. This is because the high wave number region cut by a lowpass filter operation expands and negative diffusion played by the wave number region cannot be simulated. The results suggest that it is important for reverse simulation to decide on the filter width.

\subsubsection{The Time Series of Concentration Center and Dispersion Width}

The next figures concern the concentration center $\left(X_{i c}\right)$ and dispersion width $\left(\sigma_{i}\right)$. The concentration center and dispersion width are defined by (3.1) and (3.2), respectively. Equation (3.1) explains the average location of the concentration distribution. Equation (3.2) explains the average distance from the concentration center

$$
\begin{gathered}
X_{i c}=\frac{\iiint x_{i} C\left(x_{1}, x_{2}, x_{3}\right) d x_{1} d x_{2} d x_{3}}{\iiint C\left(x_{1}, x_{2}, x_{3}\right) d x_{1} d x_{2} d x_{3}}, \\
\sigma_{i}^{2}=\frac{\iiint\left(x_{i}-X_{i c}\right)^{2} C\left(x_{1}, x_{2}, x_{3}\right) d x_{1} d x_{2} d x_{3}}{\iiint C\left(x_{1}, x_{2}, x_{3}\right) d x_{1} d x_{2} d x_{3}} .
\end{gathered}
$$

Figure 8 shows the time-lines of the concentration centers of the streamwise direction. It shows the forward analysis from 0 to 100 and the reverse analysis from 100 to 200 . 


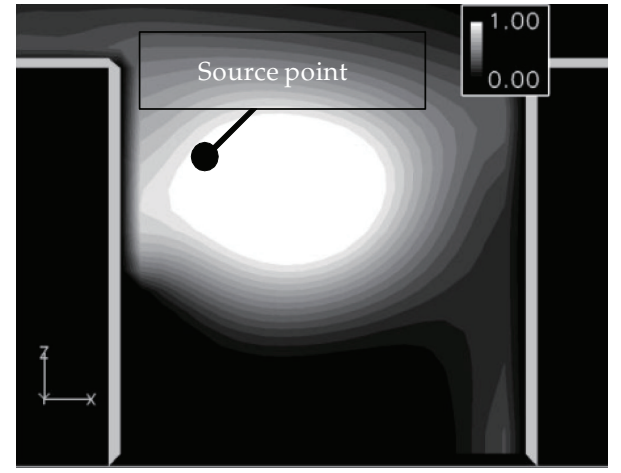

(a)

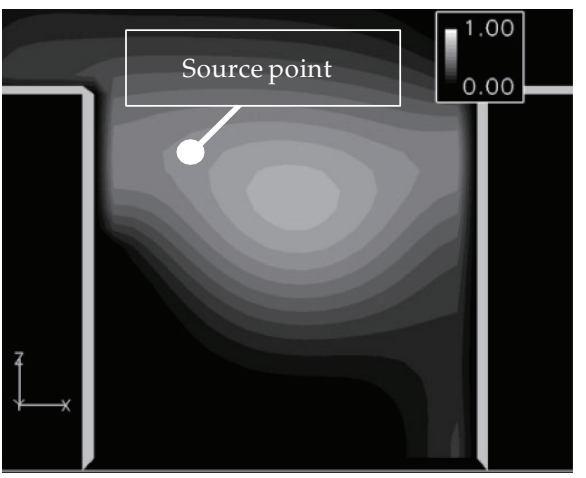

(b)

Figure 7: Reverse analysis results $\left(t U_{b} / H_{b}=200\right)$ : (a) Case 3-2 and (b) Case 3-3.

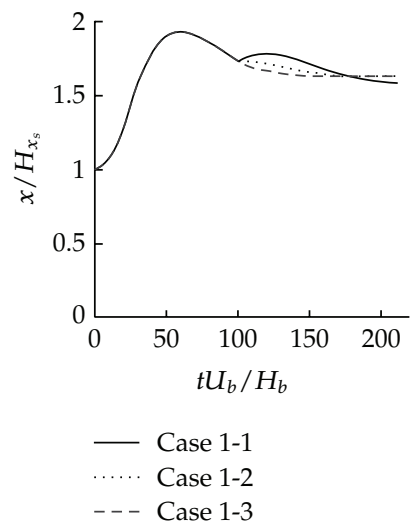

(a)

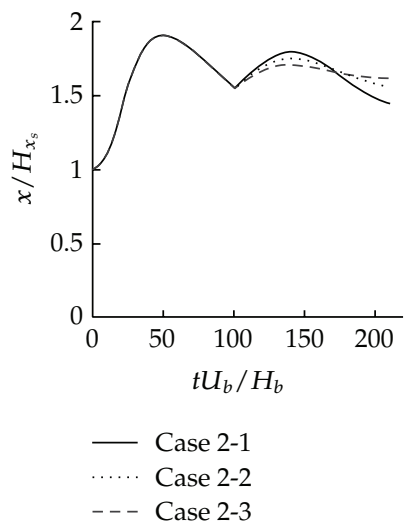

(b)

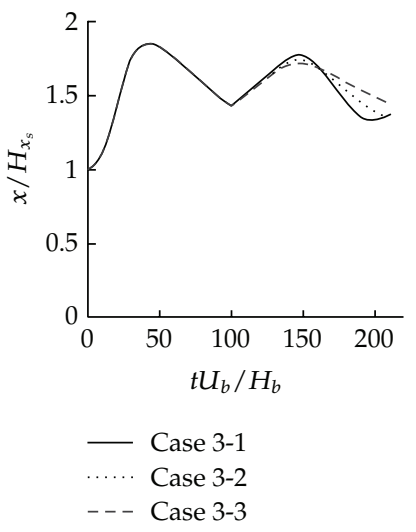

(c)

Figure 8: Time lines of concentration center of streamwise direction. (a) Case 1, (b) Case 2, and (c) Case 3.

The horizontal axes explain time that is normalized by $U_{b}$ and $H_{b}$. The vertical axes explain the concentration center that is normalized by $H_{x_{-} s}$, which is the emission point of the streamwise direction. At $t U_{b} / H_{b} \fallingdotseq 50$, the concentration centers change greatly in all cases. This is because the streamlines change greatly. When using the coarsest grid resolution (Case 1) in reverse simulation, the distribution cannot be caught. However, as the grid resolution is finer, the distribution can be perceived. In addition, we can confirm that the time-line curves become hardened with increase in filter width. In particular, the dependence on the grid resolution and filter width has a greater impact at $t U_{b} / H_{b} \fallingdotseq 150$ (corresponding to $t U_{b} / H_{b} \fallingdotseq$ 50 in forward analysis), at which the concentration centers change greatly. The great timevariation at $t U_{b} / H_{b} \fallingdotseq 150$ cannot be simulated with Case 1-3, which has the coarsest grid resolution and the broadest filter width. However, the variation can be simulated perceptively with Case 3-1, which has the finest grid resolution and the narrowest filter width.

Figure 9 shows the time lines of the concentration centers of the vertical direction. The vertical axes explain the concentration center that is normalized by $H_{z_{-} s}$, which is the emission point of the vertical direction. At $t U_{b} / H_{b} \fallingdotseq 30$, the concentration centers change greatly in all cases. This is because the streamlines change greatly as with those of the 


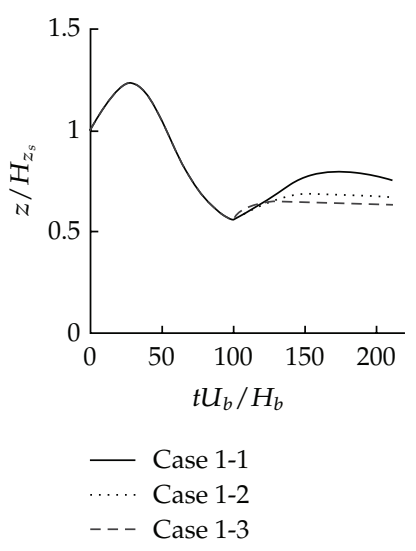

(a)

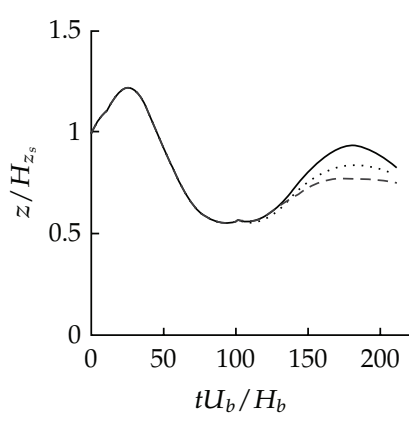

$\begin{array}{cc}\text { - Case 2-1 } \\ \text {.... } & \text { Case 2-2 } \\ \text { - - - } & \text { Case 2-3 }\end{array}$

(b)
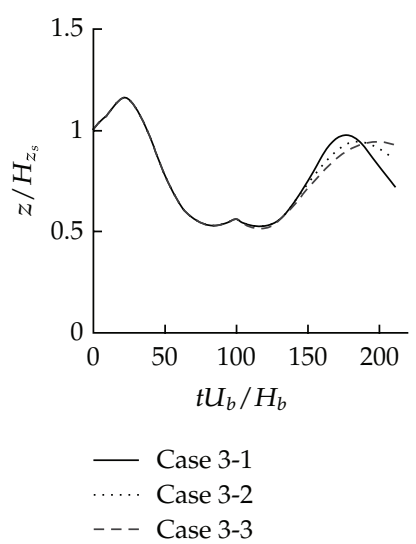

(c)

Figure 9: Time lines of concentration center of vertical direction. (a) Case 1, (b) Case 2, and (c) Case 3.

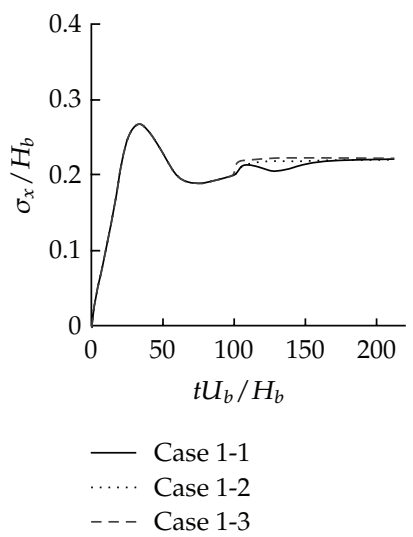

(a)

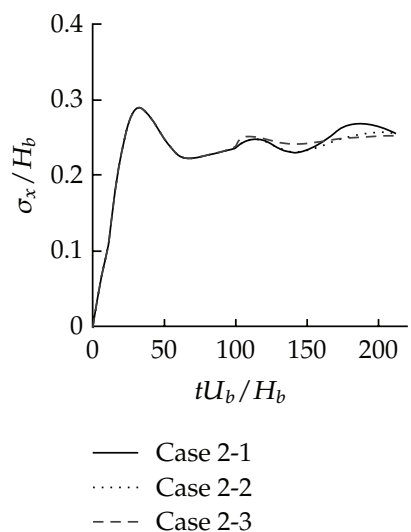

(b)

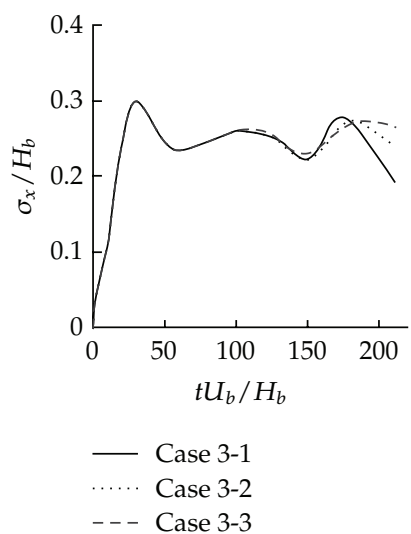

(c)

Figure 10: Time lines of concentration dispersion width of streamwise direction (a) Case 1, (b) Case 2, and (c) Case 3 .

streamwise direction. The distributions can be caught in reverse simulation, as the grid resolution is higher and the filter width is narrower. This suggests that the dependence of the reverse simulation accuracy on the grid resolution and filter width have a greater impact at $t U_{b} / H_{b} \fallingdotseq 170$ (corresponding to $t U_{b} / H_{b} \fallingdotseq 30$ in forward analysis).

Figure 10 shows the time lines of the concentration dispersion widths of the streamwise direction. We can see that the dispersion widths have maximum value at $t U_{b} / H_{b} \fallingdotseq 30$ in all cases. The distributions cannot be caught adequately by reverse analysis with Case 1 and Case 2. The analysis with Case 2-1, which has middle grid resolution, and the narrowest filter width in Case 2, can only slightly catch the distribution. Meanwhile, reverse analysis with Case 3, which has the highest grid resolution can catch the distribution exactly, especially Case 3-1. In addition, no analysis case makes the dispersion width zero at $t U_{b} / H_{b}=200$ because negative diffusion played by the high wave number is cut by the lowpass filter operation. Figure 11 shows the time lines of the concentration dispersion widths of the vertical direction. In forward analysis, the dispersion widths in all cases monotonically increase until $t U_{b} / H_{b} \fallingdotseq 50$ and remain virtually constant until $t U_{b} / H_{b}=100$. 


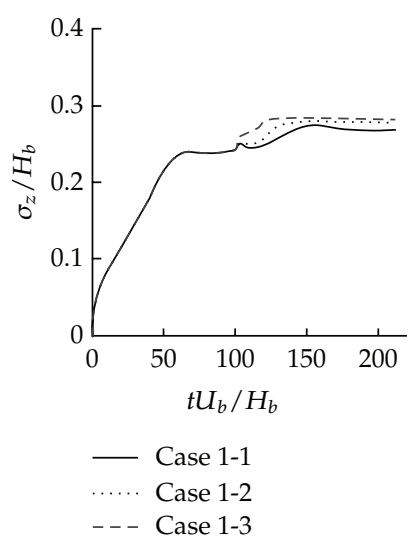

(a)

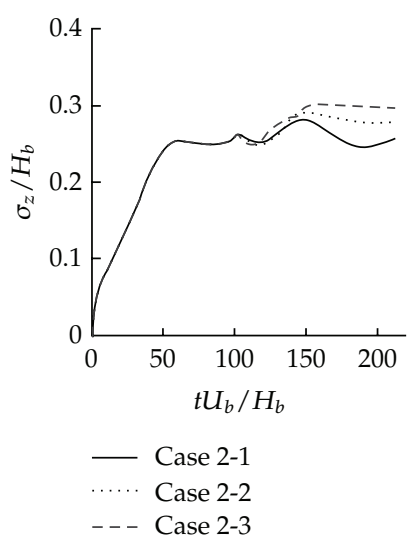

(b)

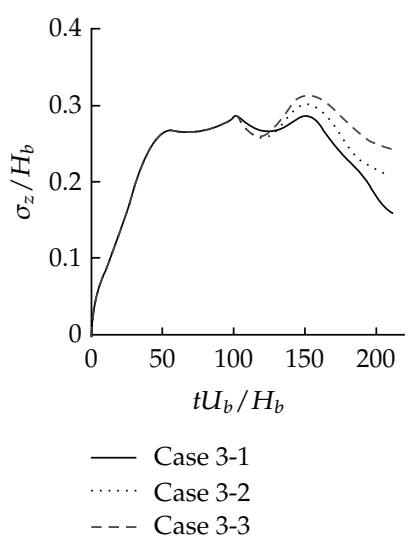

(c)

Figure 11: Time lines of concentration dispersion width of vertical direction (a) Case 1, (b) Case 2, and (c) Case 3.

Reverse simulation with Case 1 cannot catch the time series variation. Reverse simulation with Case 2 also cannot catch the negative diffusion adequately. Regarding Case 3, reverse simulation with Case 3-2 and Case 3-3 overestimate the time series variation at $t U_{b} / H_{b} \fallingdotseq 150$ (corresponding to $t U_{b} / H_{b} \fallingdotseq 50$ in forward analysis). On the other hand, the simulation with Case 3-3, which has the highest grid resolution, can precisely catch the time series variation. In addition, no analysis cases make the dispersion width zero at $t U_{b} / H_{b}=200$ as with that of the streamwise direction because negative diffusion played by the high wave number is cut by the lowpass filter operation.

Figure 12 shows the relationship between coefficients of filter operation and the distance $(\mathrm{L})$ between the concentration center and the real source point as the initial condition normalized by $H_{b}$. The horizontal axis explains the coefficient of filter operation normalized by $H_{b}$. The vertical axis explains the normalized distance. As the filter effect gets smaller, the distance shrinks. This means that the reverse simulation accuracy is more sensitive with the smaller filter effect. We can expect that reverse simulation accuracy can do better if the simulation is carried out with finer grid resolution.

As shown above, the dependence of reverse simulation on the grid resolution is stronger than that of forward analysis. In addition, the reverse simulation which uses high grid resolution is a useful method for identifying a pollutant source.

\section{Discussion}

The dependence of reverse simulation on the grid resolution and filter width is higher than that of forward analysis, as described above. The reason for this dependence is as follows.

Equation (4.1) shows a one-dimensional diffusion equation. For the sake of shorthand, the diffusion coefficient $(\Gamma)$ is a constant and positive value

$$
\frac{\partial C}{\partial t}=\Gamma \frac{\partial^{2} C}{\partial x^{2}}
$$




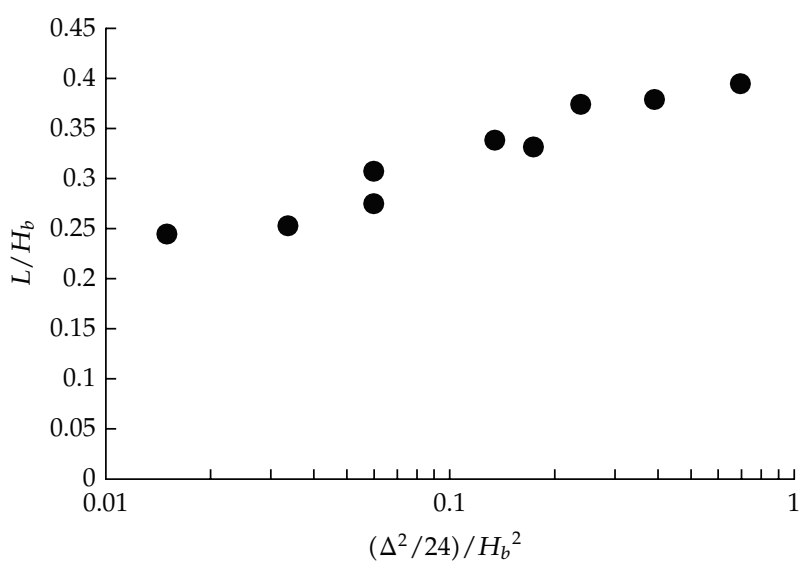

Figure 12: Relationship between coefficients of filter operation and the distance between the concentration center and the real source point in each case.

Equation (4.1) is converted into (4.3) by (4.2) (Fourier transform). $k$ shows the wave number, and $i$ shows the imaginary unit. $\widetilde{C}$ shows the concentration intensity in each wave number

$$
\begin{gathered}
C(x, t)=\int_{-\infty}^{\infty} \tilde{C}(k, t) \exp (i k x) d k_{i}, \\
\frac{\partial \tilde{C}}{\partial t}=-\Gamma k^{2} \tilde{C} .
\end{gathered}
$$

Equation (4.3) explains that $\widetilde{C}$ decays over time. Moreover, the decay effect grows strong with progressing high wave number, because the coefficient of the right-hand side of (4.3) has $-k^{2}$.

In reverse simulation, (4.3) is converted to (4.4). The sign of the right hand side of (3.2) is opposite to that of $(3.2)\left(-k^{2} \rightarrow k^{2}\right)$

$$
\frac{\partial \tilde{C}}{\partial t}=\Gamma k^{2} \tilde{C}
$$

This equation explains that $\tilde{C}$ increases over time and the increase effect in the high wave number region is stronger than that in the low wave number region. However, when analyzing the diffusion equation with $C F D$, there are rounding errors in the unresolved high wave number region. For this reason, $\widetilde{C}$ in the high wave number region grows excessively and the numerical simulation breaks down.

Next, (4.5) shows the lowpass-filtered diffusion equation by (2.8)

$$
\frac{\partial C}{\partial t}=-\Gamma\left(\frac{\partial^{2} C}{\partial x^{2}}+\frac{\Delta^{2}}{24} \frac{\partial^{4} C}{\partial x^{4}}\right)
$$




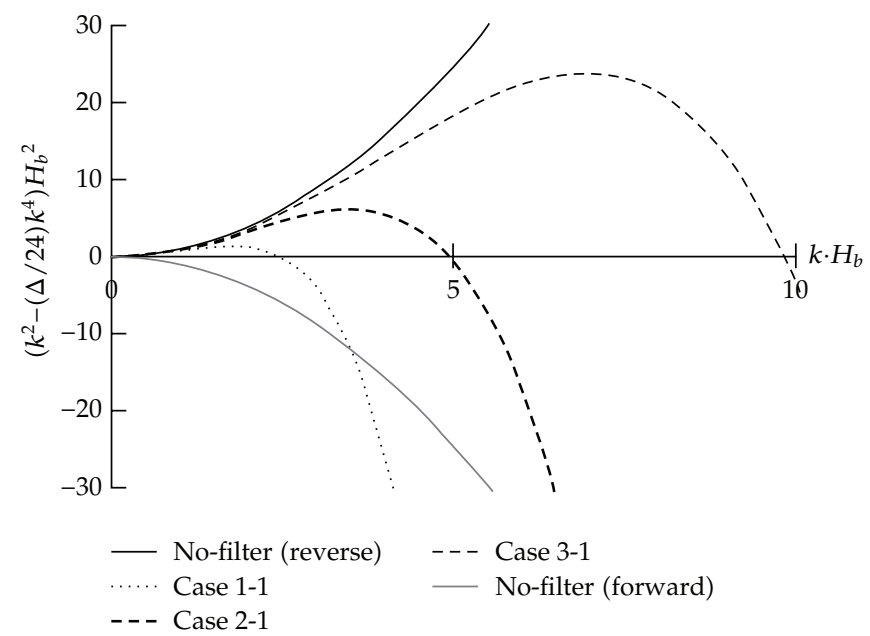

Figure 13: Coefficients of right-hand side of (4.1), (4.2), and (4.4) in each wave number (Case 1-1, Case 2-1, Case 3-1).

Equation (4.5) is converted into (4.6) by (4.2)

$$
\frac{\partial \tilde{C}}{\partial t}=\Gamma\left(k^{2}-\frac{\Delta^{2}}{24} k^{4}\right) \tilde{C} .
$$

The second term of the right-hand side added by filter operation can be seen.

Figure 13 shows coefficients of the right-hand side of (4.3), (4.4), and (4.6). The horizontal axis explains the wave number normalized by $H_{b}$, and the vertical axis explains the coefficient of the right side of the equations normalized by $H_{b}$ in each analysis case (Case 1-1, Case 2-1, Case 3-1). This figure shows the dependence of reverse simulation on the grid resolution. The figure suggests that the negative diffusion played by the low wave number can be simulated even in case of filtered equations. However, coefficients of filtered equations in the high wave number region decline. In particular, above a certain weave number, the coefficients become of negative value. This suggests that $\widetilde{C}$ in the wave number region decays over time. For this, the lowpass filter operation can suppress the increase of rounding errors and the numerical stability improves in reverse simulation. In addition, the wave number regions that negative diffusion can simulate are different in each grid resolution (see Table 2). The wave number becomes very low when using the lowest grid resolution (Case 1). Meanwhile, when using the highest grid resolution (Case 3), the simulated wave number is extended to the high wave number region.

Figure 14 shows coefficients of the right-hand side of (4.3), (4.4), and (4.6). The vertical axis explains the coefficients normalized by $H_{b}$ in each case (Case 3-1, Case 3-2, Case 3-3). This figure shows the dependence on filter width. This suggests that the wave number regions that can be simulated by negative diffusion are different in each filter width (see Table 2). When using the largest filter width (Case 3-3), the simulated wave number region becomes very low. Meanwhile, when using the narrowest filter width (Case 3-1), the simulated wave number is extended to the high wave number region.

That is the reason for the dependence of reverse simulation on the grid resolution and filter width. 


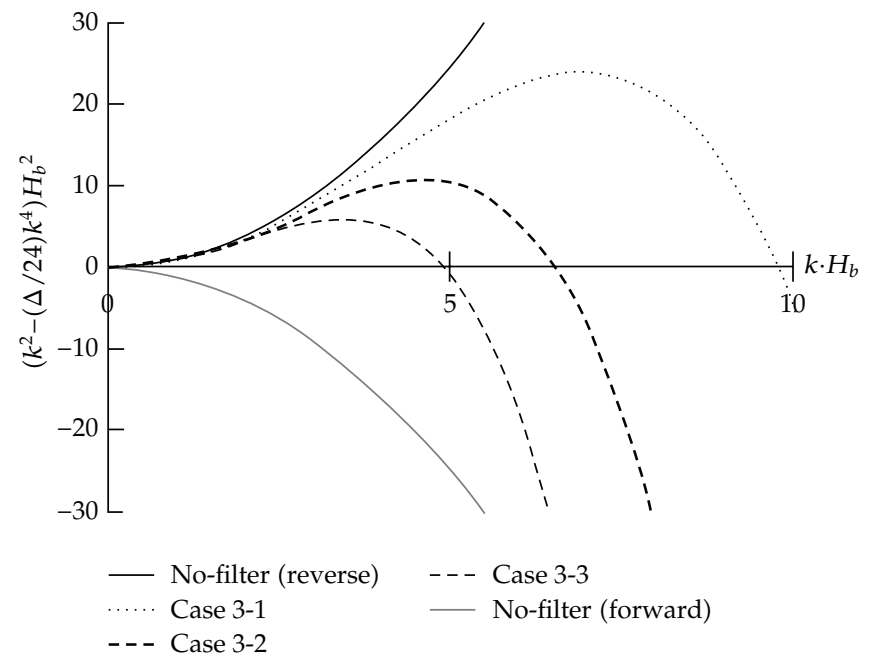

Figure 14: Coefficients of right-hand side of (4.1), (4.2), and (4.4) in each wave number (Case 3-1, Case 3-2, Case 3-3).

\section{Conclusion}

Reverse simulation is a method of identifying the source of a pollutant and is categorized as a backward method. This paper introduces the result of reverse simulation using RANS analysis in a cavity flow. With a low grid resolution or an excessively large filter width, the concentration distribution of the simulation result spreads widely. This means that the reverse simulation is less accurate and cannot be applied for identifying the pollutant source. Nevertheless, with a fine grid resolution and appropriate filter width, reverse simulation is applicable for identifying the source location.

Furthermore, this paper discusses the dependence of reverse simulation on the grid resolution and filter width with a one-dimensional diffusion equation. The simulated negative diffusion varies greatly according to the grid resolution and filter width. This is important knowledge for applying reverse simulation to practical problems.

In future, reverse simulation should be tried in various comprehensive flows and the applicability surveyed.

\section{Acknowledgment}

The present study was supported by the Kondo Jiro Grant of the Asahi Glass Foundation.

\section{References}

[1] X. Liu and Z. Zhai, "Inverse modeling methods for indoor airborne pollutant tracking: literature review and fundamentals," Indoor Air, vol. 17, no. 6, pp. 419-438, 2007.

[2] B. J. Wagner, "Simultaneous parameter estimation and contaminant source characterization for coupled groundwater flow and contaminant transport modelling," Journal of Hydrology, vol. 135, no. 1-4, pp. 275-303, 1992.

[3] A. D. Woodbury and T. J. Ulrych, "Minimum relative entropy inversion: theory and application to recovering the release history of a groundwater contaminant," Water Resources Research, vol. 32, no. 9, pp. 2671-2681, 1996. 
[4] R. M. Neupauer and J. L. Wilson, "Adjoint-derived location and travel time probabilities for a multidimensional groundwater system," Water Resources Research, vol. 37, no. 6, pp. 1657-1668, 2001.

[5] M. D. Sohn, R. G. Sextro, A. J. Gadgil, and J. M. Daisey, "Responding to sudden pollutant releases in office buildings: 1. Framework and analysis tools," Indoor Air, vol. 13, no. 3, pp. 267-276, 2003.

[6] S. M. Gorelick, B. E. Evans, and I. Remson, "Identifying sources of groundwater pollution: an optimization approach," Water Resources Research, vol. 19, no. 3, pp. 779-790, 1983.

[7] S. Alapati and Z. J. Kabala, "Recovering the release history of a groundwater contaminant via the non-linear least squares estimation," Hydrological Processes, vol. 14, pp. 1003-1016, 2000.

[8] A. N. Tikhonov and V. Y. Arsenin, Solutions of Ill-Posed Problems, Winston and Sons, New York, NY, USA, 1977.

[9] T. H. Skaggs and Z. J. Kabala, "Recovering the release history of a groundwater contaminant," Water Resources Research, vol. 30, no. 1, pp. 71-79, 1994.

[10] P. Seibert, A. Frank, and H. Kromp-Kolb, "Inverse modelling of atmospheric trace substances on the regional scale with Lagrangian models," in Proceedings of the EUROTRAC-2 Symposium, P. Midgley and M. Reuther, Eds., Garmisch-Partenkirchen, Germany, March 2002.

[11] F. Hamba, S. Abe, D. Kitazawa, and S. Kato, "Inverse problem of one-dimensional diffusion equation from the point source," Seisan Kenkyu, vol. 63, no. 1, pp. 69-73, 2011 (Japanese).

[12] T. H. Skaggs and Z. J. Kabala, "Recovering the history of a groundwater contaminant plume: method of quasi-reversibility," Water Resources Research, vol. 31, no. 11, pp. 2669-2673, 1995.

[13] S. Abe and S. Kato, "A study on improving the numerical stability in reverse simulation," Journal of Environmental Engineering, vol. 75, no. 656, pp. 891-897, 2010 (Japanese).

[14] S. Abe and S. Kato, "Study on improving numerical stability by applying filter operation to concentration flux for reverse simulation to identify pollutant source," Journal of Environmental Engineering, vol. 76, no. 662, pp. 431-438, 2011 (Japanese).

[15] M. Kato and B. E. Launder, "The modeling of turbulent flow around stationary, and vibrating square cylinder," in Proceedings of the 9th Symposium Turbulent Shear Flow, pp. 1041-1046, 1993.

[16] J. A. Viecelli, “A computing method for incompressible flows bounded by moving walls," Journal of Computational Physics, vol. 8, no. 1, pp. 119-143, 1971. 


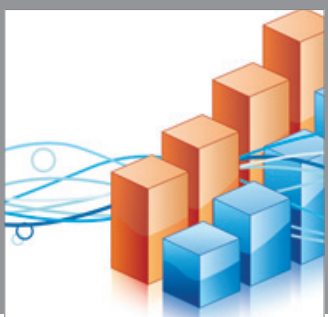

Advances in

Operations Research

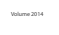

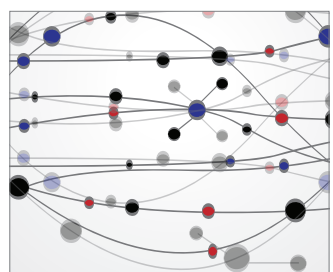

\section{The Scientific} World Journal
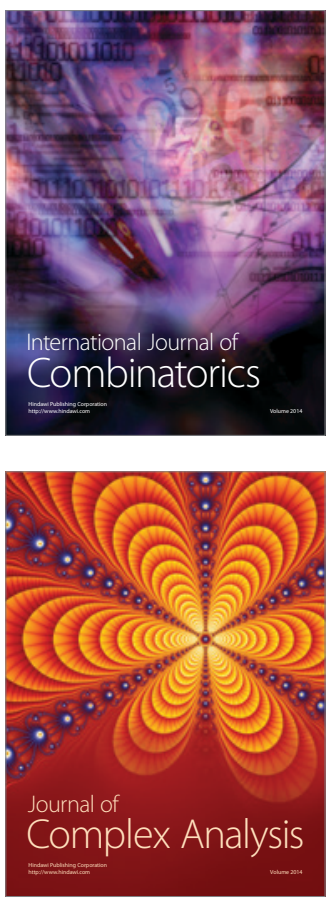

International Journal of

Mathematics and

Mathematical

Sciences
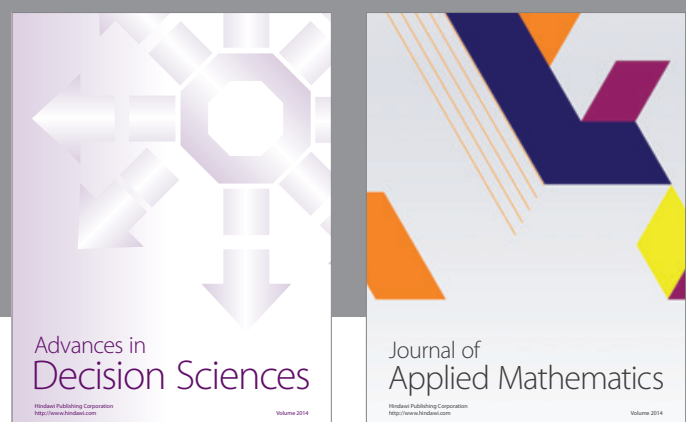

Journal of

Applied Mathematics
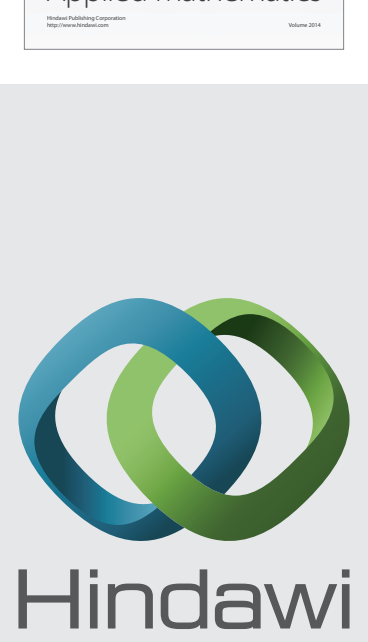

Submit your manuscripts at http://www.hindawi.com
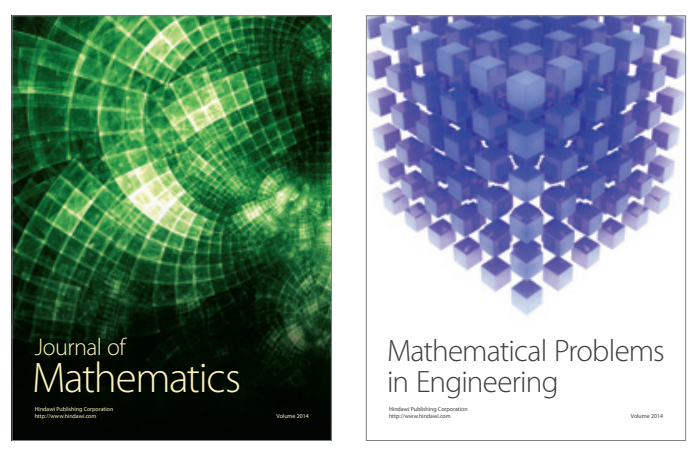

Mathematical Problems in Engineering
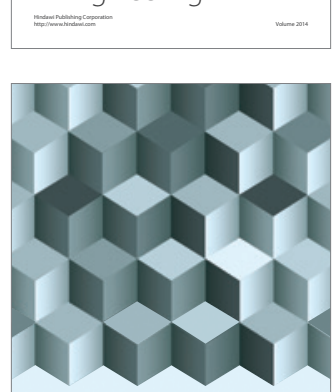

Journal of

Function Spaces
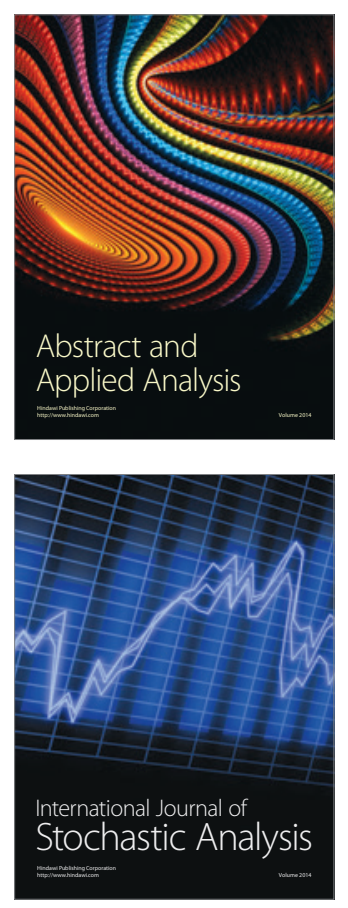

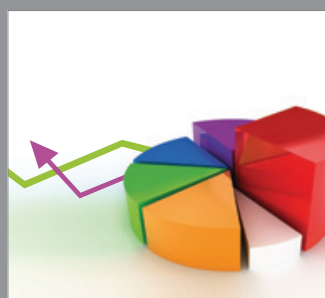

ournal of

Probability and Statistics

Promensencen
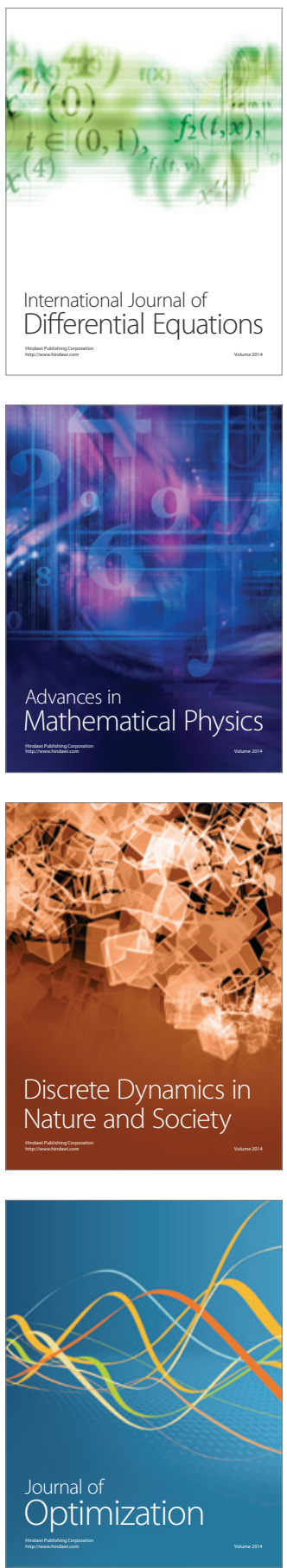